\title{
Biochemical and Structural Analysis of Hormone-sensitive Lipase Homolog EstE7: Insight into the Stabilized Dimerization of HSL-Homolog Proteins
}

\author{
Ki Hyun Nam, ${ }^{a}$ Sung-Ha Park, ${ }^{a}$ Won Ho Lee, and Kwang Yeon Hwang* \\ Division of Biotechnology, College of Life Sciences \& Biotechnology, Korea University, Seoul 136-701, Korea \\ *E-mail: chahong@korea.ac.kr \\ Received March 25, 2010, Accepted June 7, 2010
}

\begin{abstract}
Hormone sensitive lipase (HSL) plays a major role in energy homeostasis and lipid metabolism. Several crystal structures of HSL-homolog proteins have been identified, which has led to a better understanding of its molecular function. HSLhomolog proteins exit as both monomer and dimer, but the biochemical and structural basis for such oligomeric states has not been successfully elucidated. Therefore, we determined the crystal structure of HSL-homolog protein EstE7 from a metagenome library at 2.2 Å resolution and characterized the oligomeric states of EstE7 both structurally and biochemically. EstE7 protein prefers the dimeric state in solution, which is supported by its higher enzymatic activity in the dimeric state. In the crystal form, EstE7 protein shows two-types of dimeric interface. Specifically, dimerization via the external $\beta 8$-strand occurred through tight association between two pseudosymmetric folds via salt bridges, hydrogen bonds and van der Waals interactions. This dimer formation was similar to that of other HSL-homolog protein structures such as AFEST, BEFA, and EstE1. We anticipate that our results will provide insight into the oligomeric state of HSLhomolog proteins.
\end{abstract}

Key Words: EstE7, HSL-homolog protein, Dimerization, Oligomeric state, Crystal structure

\section{Introduction}

Fatty acids that are released as a result of HSL (hormone sensitive lipase)-and MGL (monoglyceride lipase)-catalyzed lipolysis are exported to the circulatory system and eventually provide energy to other tissues. Because fatty acids are the major energy source in mammals, HSL plays a major role in energy homeostasis and metabolism. In addition to this pivotal role, HSL may play several important roles in other tissues in which it is expressed. The majority of HSL expression is observed in white adipose tissue; however, significant expression is also observed in muscle, the testis, adrenal glands, brown adipose tissue, mammary glands, the pancreas and other tissues in which HSL likely plays more restricted, localized roles. The primary enzymatic activities of HSL are in the formation of triacyl and diacyl glycerol, cholesteryl, steroids and retinyl ester hydrolysis. $1,2,3$

Quantitatively, adipose tissue triacylglycerol is the most important energy store in mammals. Hydrolysis of this triacylglycerol is tightly controlled by a variety of hormonal and neural influences that allow the release of this energy to be regulated in response to the changing energy requirements of the animal. The key enzyme involved in this release (lipolysis) is hormonesensitive lipase (HSL) (EC 3.1.1.3). ${ }^{2}$ Mobilization of fatty acids from rat and human fat cells is selective according to the molecular structure and carbon atom chain length. This study was conducted to determine if the release of individual fatty acids from triacylglycerols (TAG) by hormone-sensitive lipase (HSL) plays a role in the selectivity of fatty acid mobilization. ${ }^{3}$ The structural and biochemical characteristics of several HSL homo$\log$ proteins isolated from bacteria or uncultured bacterium have been reported, including EstE5 and EstE7 from uncultured

${ }^{\mathrm{a}}$ These authors contributed equally to this article. bacterium, EST2 from Alicyclobacillus acidocaldarius, EstE1 from an uncultured archaeon, AFEST from Archaeoglobus fulgidus and brefeldin A esterase from Bacillus subtilis. ${ }^{4-11}$ These proteins all contain the N-terminal regulatory (CAP) and C-terminal catalytic (CAT) domain. In addition, the catalytic triad by charge-relay system of Ser-His-Glu/Asp, in which each residue acts as a nucleophile, proton carrier and charge relay, is conserved among these proteins. Thus, these proteins share a common structural configuration, even though they exist as dimers or monomers in solution. As a result, the molecular function of these proteins has not been successfully elucidated until now. ${ }^{4,6,9}$

We recently reported the biological and structural analysis of EstE7 from a metagenome library, ${ }^{9}$ in which EstE7 was eluted as both a monomer and dimer upon size exclusion chromatography. For crystallization, we used the monomeric protein, but the crystal structure at a resolution of $2.8 \AA$ revealed a dimeric arrangement in the asymmetric unit. Accordingly, we considered dimer formation for the crystallographic results, but this information also did not enable successful elucidation of the molecular characteristics of this molecule. ${ }^{9}$ In this study, we determined the crystal structure of EstE7 at a resolution of 2.2 A. We also investigated the oligomeric states of EstE7 by size exclusion chromatography and dynamic light scattering, combined with an enzyme activity assay. Structural comparison with other HSL-homolog proteins revealed that HSL proteins show various oligomeric states with no biological correlation. The results presented here will provide useful structural information for future studies.

\section{Materials and Methods}

Protein preparation and size determination. EstE7 (Accession no. Q0GMU1) was cloned, purified and crystallized from the metagenome library as previously described. ${ }^{9}$ Briefly, after 
Table 1. Data collection and refinement statistics for EstE7

\begin{tabular}{lc}
\hline Data collection statistics & $I 2{ }_{1} 2_{1} 2_{1}$ \\
Space group & $\mathrm{a}=117.157, \mathrm{~b}=127.699$, \\
Unit cell parameters $(\AA)$ & $\mathrm{c}=232.870$ \\
Wavelength $(\AA)$ & 1.230 \\
Resolution range $(\AA)$ & $50.0-2.2(2.28-2.20)$ \\
Measured reflections & 420468 \\
Unique reflections & 84721 \\
Completeness & $95.6(82.5)$ \\
Redundancy & $5.0(3.0)$ \\
Average $\mathrm{I} / \sigma(\mathrm{I})$ & $11.1(1.83)$ \\
$\mathrm{R}_{\text {merge }}(\%)^{a}$ & $9.5(33.2)$ \\
\hline Refinement statistics & \\
${\text { Resolution }(\AA)^{b}}^{b}$ & $48.26-2.20$ \\
Reflections in working set & 80451 \\
Reflections in test set $^{c}$ & 4253 \\
$\mathrm{R}_{\text {work }} / \mathrm{R}_{\text {free }}(\%)^{c}$ & $22.26 / 28.22$ \\
r.m.s.d. bonds $(\AA)$ & 0.020 \\
r.m.s.d. angles $\left({ }^{\circ}\right)$ & 1.976 \\
\hline Ramachandran plot $(\%)^{d}$ & \\
favored $_{\text {allowed }}$ & 94.6 \\
\hline
\end{tabular}

${ }^{a} \mathrm{R}_{\text {merge }}=\sum_{h} \sum_{i}|I(h, i)-<\mathrm{I}(h)>| / \sum_{h} \sum_{i} I(h, i)$, where $I(h, i)$ is the intensity of the $i$ th measurement of reflection $\mathrm{h}$ and $<\mathrm{I}(h)$ is the mean value of $I(h, i)$ for all i measurements. ${ }^{b}$ Refinement resolutions were recalculated by Sfcheck. ${ }^{c} \mathrm{R}_{\text {work }}=\sum|| F_{\text {obs }}|-| F_{\text {calc }}|| / \sum\left|F_{\text {obs }}\right|$, where Fobs and Fcalc are the observed and calculated structure-factor amplitudes, respectively. $R_{\text {free }}$ was calculated as $\mathrm{R}_{\text {work }}$ using a randomly selected subset of $\sim 5 \%$ of the unique reflections not used for structure refinement. ${ }^{d}$ Categories were defined by MolProbity.

the His Trap purification step, the EstE7 protein were further purified on a Hi-Load 26/60 Superdex 200 prepgrade column (GE healthcare) in buffer composed of $10 \mathrm{mM}$ Tris- $\mathrm{HCl}(\mathrm{pH}$ 8.0) and $50 \mathrm{mM} \mathrm{NaCl}$. Based on the gel filtration results, the eluted peak samples were evaluated for esterase activity. In addition, the eluted peaks were analyzed by dynamic light scattering (DLS) to confirm the oligomeric state. Prior to the DLS experiment, aggregated, dimer and monomer EstE7 proteins were concentrated at 1,3 and $3 \mathrm{mg} / \mathrm{mL}$, respectively. DLS measurements were performed using the DYNAPRO TITAN dynamic light scattering systems (Wyatt Technologies) at $25^{\circ} \mathrm{C}$ and data were analyzed using the DYNAMICS (version 6.9.2.9) software provided by the manufacturer.

Enzyme activity. The enzyme activity of the EstE7 proteins in various oligomeric states was measured using $p$-nitrophenyl butyrate. ${ }^{12}$ The esterase activity was measured by spectrophotometric assay at $\mathrm{pH} 7.5$ and $30^{\circ} \mathrm{C}$ for 5 min using $5 \mathrm{mM}$-nitrophenyl butyrate as the substrate. Larger aggregates (void volume in size exclusion chromatography), aggregated, dimer and monomer EstE7 proteins were concentrated to $3 \mathrm{mg} / \mathrm{mL}$ and then used for subsequent analyses. The $p$-nitrophenol released from the ester substrates was monitored by measuring the absorbance at $350-450 \mathrm{~nm}$. The enzyme activity was determined based on the absorbance at $400 \mathrm{~nm}$.

Crystallization, data collection and structure determination. The purified protein was concentrated to $30 \mathrm{mg} / \mathrm{mL}$ and suitable crystals for X-ray diffraction were then obtained with $0.1 \mathrm{M}$ Bis-Tris propane at $\mathrm{pH}$ 7.0, 0.2 M ammonium sulfate and $1 \mathrm{M}$ lithium sulfate using the hanging-drop vapor-diffusion method at $22{ }^{\circ} \mathrm{C}$. X-ray diffraction data were collected at beamline $6 \mathrm{C}$ using the Pohang Light Source (PLS, Korea). Crystals were flash-frozen in a liquid nitrogen stream using $25 \%$ (v/v) glycerol or ethylene glycol as a cryoprotectant. Both solutions revealed nearly identical X-ray diffraction patterns. The diffraction data were then processed and scaled using the HKL2000 program. ${ }^{13}$ The initial phases were obtained using molecular replacement. The MOLREP program within the CCP4 program suite was employed along with a model of EstE7 (PDB code 3DMN). ${ }^{9} 14$ The structure was refined using restrained refinement without TLS refinement from the Refmac5, ${ }^{15}$ after which the refinement resolutions were recalculated by Sfcheck. ${ }^{16}$ The model was adjusted using the WinCoot program, ${ }^{17}$ and the final models were validated with MolProbity. ${ }^{18}$ Structural analyses were conducted using the $\mathrm{CCP} 4$ program, CNS program, PISA and DaliLite sever. ${ }^{9-22}$ Molecular graphic figures were generated using PyMOL (www.pymol.org). The data collection and structural refinement statistics are shown in Table 1. The coordinates and structural factors for EstE7 from the metagenome library have been deposited in the Protein Data Bank under accession code $3 \mathrm{~K} 6 \mathrm{~K}$.

\section{Results and Discussion}

Oligomeric state and enzyme activity of EstE7. Size exclusion chromatography through HiLoad 26/60 Superdex 200 revealed that EstE7 (36 kDa, including the expression tag) at an initial concentration of $30 \mathrm{mg} / \mathrm{mL}$ was eluted in three peaks, which reflected the presence of an aggregated $(400 \mathrm{kDa})$, dimeric $(75 \mathrm{kDa})$ and monomeric $(40 \mathrm{kDa})$ form (Fig. 1A). The enzyme activities of the EstE7 proteins in different oligomeric states (No. 2, 3 and 4 in Fig 1A; including the void volume sample as No.1) were measured on a spectrophotometer within 24 hours of elution using $p$-nitrophenyl butyrate as a model substrate. We found that all of the oligomeric states of EstE7 exhibited esterase activity, with the activity of highly aggregated, aggregated, dimeric and monomeric EstE7 proteins being approximately $20,15,100$ and $40 \%$, respectively (Fig. 1B). Interestingly, the dimeric EstE7 protein showed a higher enzyme activity than proteins in the other oligomeric states. Although we have not found clear evidence of the high activity of the dimer form of the protein based on biological studies, the EstE7 activity observed here is similar to that of the HSL protein, in which dimeric HSL has an activity 40-fold greater than that of the monomeric HSL protein. ${ }^{23}$ Based on several purification batches (data not shown), we found that the EstE7 proteins eluted atypical elution sizes and that aggregated, dimeric and monomeric EstE7 proteins had various ratios of elution peaks upon size exclusion chromatography. We conducted DLS experiments to confirm the accuracy of the size of the eluted EstE7 proteins as aggregated, dimer and monomer proteins from the purification batch used to generate Fig. 1A (No 2, 3 and 4 in Fig. 1A). DLS analysis indicated that all three samples, which were in different oligomeric states following size exclusion chromatography, had the same molecular weight with the dimeric 
(A)

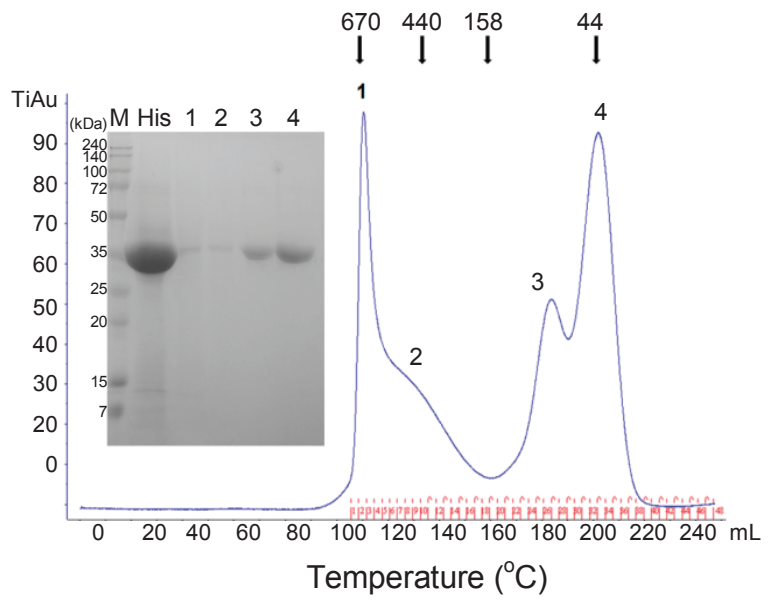

(B)



Figure 1. Analysis of the oligomeric state of EstE7 (A) Size determination using gel filtration chromatography. $0.83 \mathrm{mM}$ protein solution was applied to a HiLoad 26/60 Superdex 200 column that was pre-equilibrated with buffers containing $10 \mathrm{mM}$ Tris-HCl at $\mathrm{pH} 7.0$ and $50 \mathrm{mM} \mathrm{NaCl}$. Numbers 1, 2, 3 and 4 indicate the void, aggregated, dimer and monomer size. In the SDS-PAGE gel, 'His' indicates the sample injected before size exclusion chromatography. (B) The relative activity of the EstE7 protein measured at $400 \mathrm{~nm}$ using a spectrophotometer to evaluate the various oligomeric states. All oligomeric states showed esterase activity.

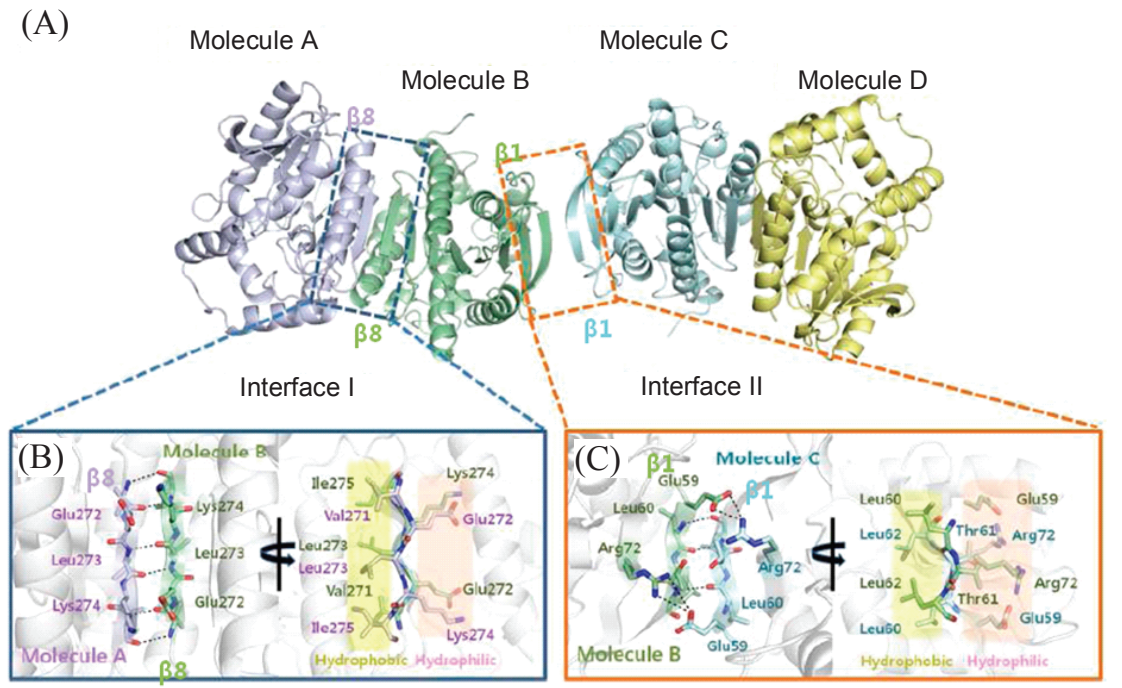

Figure 2. Crystal structure of EstE7 (A) EstE7 molecules in the asymmetric unit. Each molecule in the asymmetric unit has an identical overall structure with an r.m.s. deviation of approximately $0.15 \AA$. (B) Interface I between molecules A and B (or, C and D). Analysis revealed that approximately $14.8 \%$ of the monomer surface area was buried, and that it was stabilized by hydrophobic, hydrophilic and van der waals interactions. Both molecules form the five main chain (hydrogen bond) interactions in $\beta 8$-strands. In addition, hydrophobic and hydrophilic residues are arranged into up and down of strands. (C) Interface II between molecules B and C. Analysis revealed that approximately $4.4 \%$ of the monomer surface area was buried, and that it was stabilized by hydrophobic and hydrophilic interactions. Both molecules formed three main chain (hydrogen bond) interactions in $\beta 1$-strands. Based on the structure, the dimer interface between molecules A and B is more stable than the interface between molecules $\mathrm{B}$ and $\mathrm{C}$.

(60 - $80 \mathrm{kDa}$ ) (data now shown). These DLS results were not consistent with the size exclusion chromatography results of the aggregated form and monomer form of the EstE7 protein. It has been reported the aggregated form of EstE7 may exist in a self-association/aggregation state at high concentrations. ${ }^{24}$ Based on our findings, the aggregated form of EstE7 is capable of dissociation and subsequent dimer formation; thus, the aggregated form is a temporary state. However, the monomer form of EstE7 is more likely to undergo dimerization because EstE7 is thermodynamically stabilized based on the structure (discussed below). Taken together, our results revealed an unusual oligomeric state of EstE7 in solution. EstE7 proteins prefer the dimeric formation, which results in the aggregated form dissociating into the dimer form under diluted conditions, and monomer forms eventually forming dimers under natural conditions. This is the first report of the reversible oligomeric state of EstE7, and we believe that this biological observation will enable a better understanding of the oligomeric state of HSL-homolog proteins.

Stabilized dimenization of EstE7. We determined the crystal structure of EstE7 at a resolution of $2.2 \AA$, which extends the resolution of $2.8 \AA$ that was used in a previous study. ${ }^{9}$ The EstE7 protein features an $\alpha / \beta$-hydrolase fold consisting of nine $\alpha$-helices, an eight-stranded $\beta$-sheet and several long loops. The protein 
Table 2. Structural alignment with other HSL-homolog proteins

\begin{tabular}{|c|c|c|c|c|c|c|}
\hline Portein name & Strains & Z-score & r.m.s.d. & Aligned residues & Identity (\%) & PDB code \\
\hline EstE5 & Uncultured bacterium & 45.8 & 1.6 & 293 & 38 & 3FAK \\
\hline EST2 & A. acidocaldarius & 38.7 & 2.1 & 291 & 28 & 1QZ3 \\
\hline EstE1 & Uncultured archaeon & 38.5 & 1.8 & 276 & 25 & $2 \mathrm{C} 7 \mathrm{~B}$ \\
\hline AFEST & A. fulgidus & 38.3 & 2.1 & 290 & 25 & $1 \mathrm{JJI}$ \\
\hline BFAE & B. subtilis & 33.8 & 2.2 & 281 & 21 & 1JKM \\
\hline
\end{tabular}

consists of a CAP (regulatory) and a CAT ( $\alpha / \beta$ fold; catalytic) domain. This structure has an overall structure identical to that of a previously reported EstE7 structure with an r.m.s. deviation of approximately $0.15 \AA$ for $294 \mathrm{C} \alpha$ (Met 16-Ser309). In the crystallographic packing, four molecules in the asymmetric unit of EstE7 were found to have a nearly identical conformation, which upon superposition results in a $\mathrm{C} \alpha$ r.m.s. deviation of $1.0 \AA$. In the asymmetric unit, each EstE7 protein formed three buried surface regions, and two types of dimerization interface were observed. The two dimers were found to be related by a non-crystallographic two-fold pseudosymmetry (Fig. 2A). The first dimerization interface (Interface I in Fig. 2A) is located between molecules $\mathrm{A}$ and $\mathrm{B}$ and is similar to the interface between molecules $\mathrm{C}$ and $\mathrm{D}$ with an r.m.s. deviation of $0.15 \AA$ for $294 \mathrm{C} \alpha$ (Met16-Ser309). In dimeric interface I, the $\beta 8$ strands in both molecules A and B play a key role in the tightly associated dimer formation. Six residues (Val271, Glu272, Leu273, Lys274, Ile275, and Trp276) are involved in the dimerization (Fig. 2B). This dimer formation by interface I results in an extensive interface that buries approximately $1,800 \AA^{2}$ (14.8\%) of the total monomer surface area of $12,200 \AA^{2}$. Among these interactions, both $\beta 8$-strands in molecules $\mathrm{A}$ and $\mathrm{B}$ formed an antiparallel association via the $\beta 8$-strand that was stabilized by six hydrogen bonds of the main chain of Val271, Leu273 and Ile275 within $2.8 \sim 3.4 \AA$. Moreover, the hydrophobic (Val271, Leu273 and Ile275) and hydrophilic (Glu272 and Lys274) residues are located along different faces in the $\beta 8$-strand. Thus, dimerization of the EstE7 between molecules A and B is strongly stabilized by several interactions based on the structure. The second dimerization interface (Interface II in Fig. 2A) is located between molecules $\mathrm{B}$ and $\mathrm{C}$. This dimer formation was formed by antiparallel association of the $\beta 1$-strands (Fig. 2C) and formed a less tightly associated dimer than the interface between molecules A and B (or molecules C and D). This dimer formation results in an interface that buries approximately $540 \AA^{2}(4.4 \%)$ of the $12,200 \AA^{2}$ total monomer surface area. Among the dimer interface residues of molecules $B$ and $C$ of EstE7, the amino- and carboxyl group of the main-chain in Leu60, Thr61 and Leu62 formed the short $\beta$-strand within 2.7 $3.2 \AA$. Moreover, both Glu59 and Arg72 formed a salt bridge of approximately $3.2 \AA$. Taken together, molecules A-B (or C-D) have a larger buried surface region than molecules B-C, and also contain preferred amino-acid arrangements based on the structure analysis. Hydrophobic or hydrophilic interactions are distributed in both dimerization interfaces; thus, the dimer formation of EstE7 indicates that there are salt bridges, hydrogen bonds and van der Waals interactions. Conversely, evaluation of the interface energy of Interface I and II using the interface
(A)
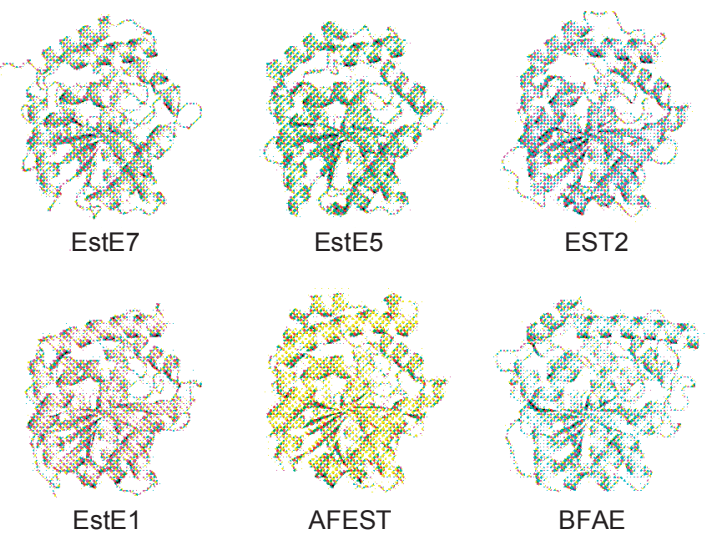

(B)
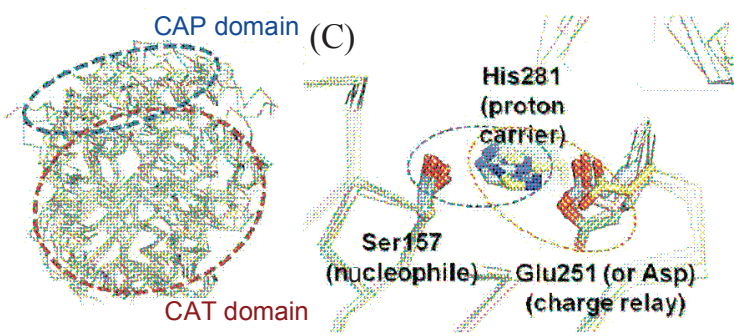

Figure 3. Structural comparison of HSL-homolog proteins. (A) Monomer structure of HSL-homolog proteins. All structures contain the CAP- and CAT- domain. (B) Superimposition of the HSL-homolog proteins. The EstE7 proteins were structurally similar with a Z-score and r.m.s. deviation of $>35$ and $<2.5$, respectively. (C) Superimposition of the catalytic triad of HSL-homolog proteins. The charge relay system in EstE7 and EstE5 utilizes glutamic acid, whereas other HSL-homolog proteins utilize aspartic acid. Although charge related residues have different amino acid sequences, they have the same molecular function.

calculation through the PISA server revealed solvation free energies $\left(\Delta^{\mathrm{i}} \mathrm{G}\right)$ of approximately -10.0 and $-0.6 \mathrm{kcal} / \mathrm{mol}$, respectively. ${ }^{21}$ These findings suggest that A-B formation may be the preferred dimer formed by EstE7 in solution, and that it will be highly stabilized based on its structure.

Structural comparison with HSL homolog proteins. A search for structural homologues of EstE7 using the DALI program identified several candidate proteins. Among these structures, we selected five HSL-homolog proteins, EstE5 from an uncultured bacterium, EST2 from Alicyclobacillus acidocaldarius, EstE1 from an uncultured archaeon, AFEST from Archaeoglobus fulgidus and brefeldin A esterase from Bacillus subtilis. ${ }^{4-11}$ Detailed structural alignment results are shown in Table. 2. The CAP and CAT domains were conserved among structures, which is supported by Z scores and r.m.s. deviations of $>35$ and $<2.5$, respectively (Fig. 3A and 3B). Each of these proteins also contains a typical charge-relay system in the catalytic triad, 

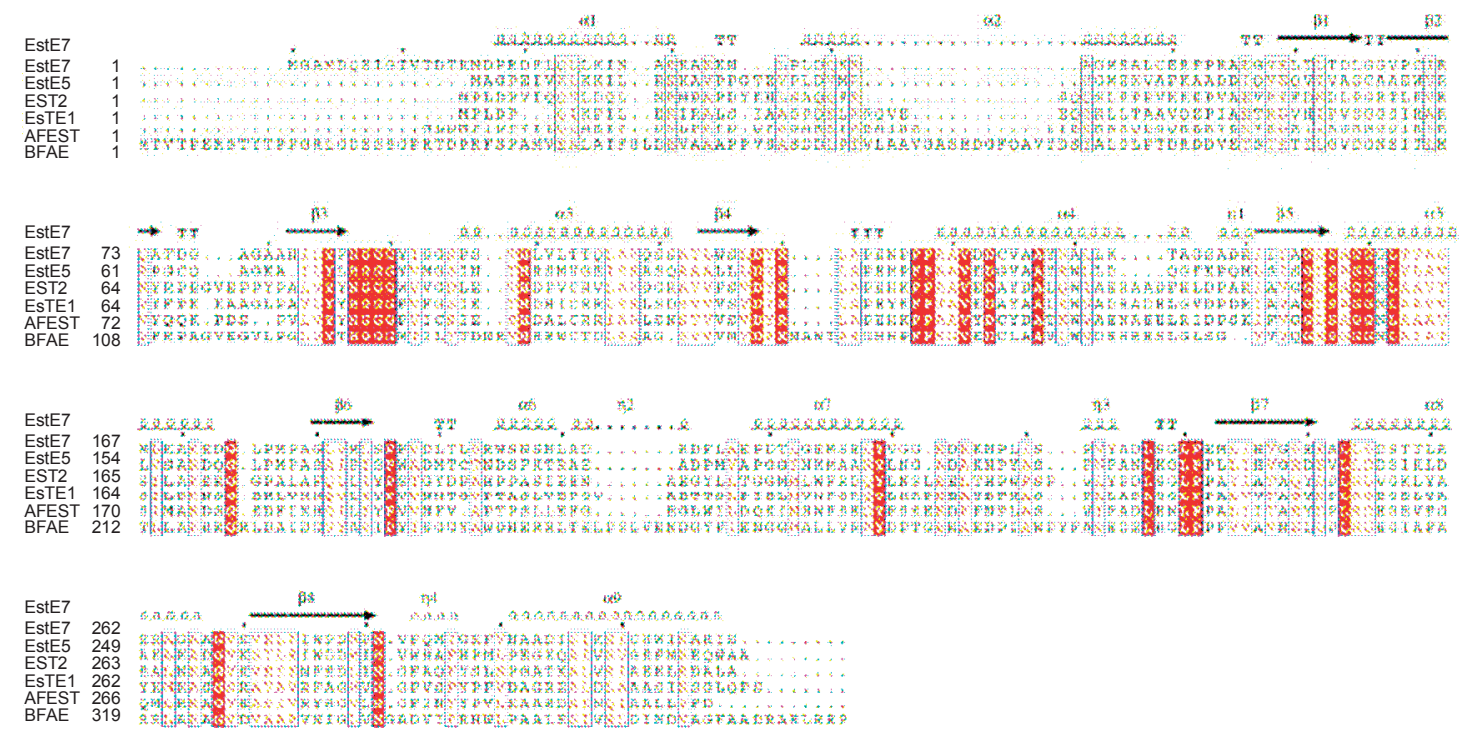

Figure 4. Sequential and structural comparisons with other members of the HSL family. (A) Representative sequences from a multiple alignment of the EstE7 (Q0GMU1) sequence using CLUSTAL W. The three sequences shown are from the SWISSPROT database: EstE5 is from the metagenome library (Q0GMU2), carboxylesterase is from an uncultured archaeon (Q5G935), EST2 is from Alicyclobacillus acidocaldarius (Q7SIG1), AFEST is from Archaeoglobus fulgidus (O28558) and BFAE is from Bacillus subtilis (O68884).

(A)

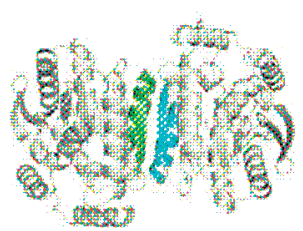

(C)

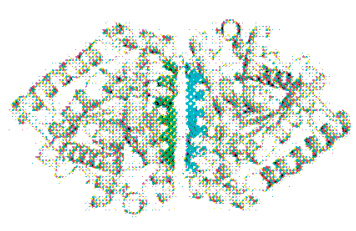

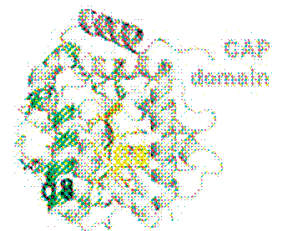

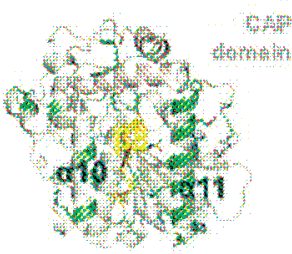

(B)

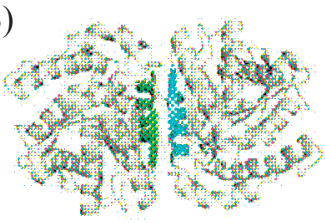

(D)

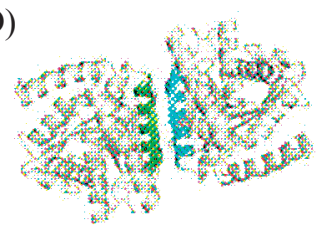

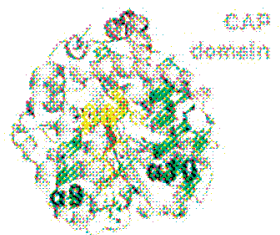

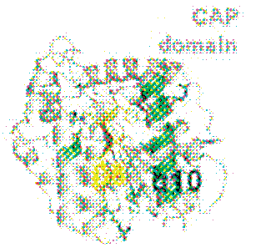

Figure 5. Dimer formation of HSL-homolog proteins. (A) EstE7 (B) EstE1 (C) AFFEST and (D) BFAE showed dimer formation in the crystal structure and the solution state. These HSL-homolog proteins produced similar dimer forms using the edge $\beta 8$-strand for dimerization. The overall dimer forms of these proteins were similar; however, each monomer had surface regions that were buried by different secondary structures. The $\alpha$-helix and $\beta$-strand in the buried surface in the dimer formation are colored green and yellow, respectively.

although some proteins contain a different charge relay residue on the catalytic triad (glutamate or aspartate). Nevertheless, both combinations maintain a functional charge-relay system (Fig. 3C) ${ }^{9,10}$ The EstE5 and EST2 proteins exist as monomers in solution, which was confirmed by crystallographic biochemical tests. Based on the results of the structural analysis of EstE7, the $\beta 1$ - and $\beta 8$-strands of EstE5 and EST2 were also exposed in solution; however, in these two proteins the strands had different sequences than in EstE7. Specifically, they consisted of a random-mixture of hydrophobic and hydrophilic residues when compared with EstE7 (Fig 4). These findings suggest that EstE5 and EST2 do not prefer dimerization induced by their $\beta 1$ - or $\beta 8$-strands in solution. Recently, we evaluated the structural and biological characteristics of EstE5, and we did not detect the dimeric species of EstE5 in solution, which is consistent with our interpretation above.$^{10}$ Conversely, the AFEST, BFAE and EstE1 proteins exist as dimers in solution and in the crystal
(Fig. 5). The results of detailed structural analysis of the buried residues in the dimer interface of the EstE7 homologues are shown in Table 3. Additionally, the dimer formations of the EstE7, AFEST, BFAE and EstE1 proteins were commonly stabilized by salt bridges, hydrogen bonds and van der Waals interactions. The dimer interfaces of those proteins show low sequence homology, as well as dimer conformations with unique configurations (Fig. 4 and Fig. 5). In previous studies, AFEST and BFAE were reported to be active as monomers in solution, and both had active sites that remained fully accessible to the substrate, indicating that their dimerization should not be related to catalytic function. ${ }^{4,6}$ In the EstE7 structure, dimerization interfaces of EstE7 are separated by the substrate entrance pore (CAP domain) and catalytic triad (CAT domain) by a distance of approximately 20 - $30 \AA$. This structure suggests that the molecular function of the dimer will not affect the catalytic activity of EstE7 because the CAP domain and active sites of 
Table 3. Residues in buried-surface of dimer formation of HSL-homolog proteins

\begin{tabular}{|c|c|c|}
\hline & Interaction & Residues in buried-surface of the dimer formation \\
\hline \multirow{2}{*}{$\begin{array}{l}\text { EstE7 } \\
(\mathrm{A}-\mathrm{B})\end{array}$} & Hydrophobic (12) & Trp195, Leu254, Ala261, Gly265, Ala266, Gly268, Val269, Va1271, Leu273, Ile275, Asp276, Pro277 \\
\hline & Hydrophobic (10) & Arg203, Ser249, Ser255, Thr258, Glu262, Arg263, Ser270, Glu272, Lys274, Asp278 \\
\hline \multirow{2}{*}{ AFEST } & Hydrophobic (16) & $\begin{array}{l}\text { Pro5, Pro8, Va19, Leu108, Pro246, Leu248, Ala275, Ile277, Val278, Val293, Lys295, Ala296, Ala306, } \\
\text { Leu307, Val309, Phe310 }\end{array}$ \\
\hline & Hydrophobic (17) & $\begin{array}{l}\text { Asp7, Arg104, Arg107, His183, Glu262, Gln266, Arg269, Glu274, Ser276, Arg279, Tyr280, Arg281, } \\
\text { Arg298, Asp299, Asn302, Gln303, Asp311 }\end{array}$ \\
\hline \multirow{2}{*}{ BFAE } & Hydrophobic (18) & $\begin{array}{l}\text { Pro27, Pro31, Leu246, Pro250, Val301, Ile315, Ala322, Val328, Ala329, Ala330, Val332, Ile334, Pro349, } \\
\text { Ala350, Ala351, Gly361, Phe362, Leu369 }\end{array}$ \\
\hline & Hydrophobic (17) & $\begin{array}{l}\text { Arg28, Ser30, Thr84, Arg86, Glu306, Arg319, Arg323, Asp327, Arg331, Asn333, Glu353, Ser354, Arg357, } \\
\text { Asp358, Asp365, Arg366, Arg368 }\end{array}$ \\
\hline \multirow{2}{*}{ EstE1 } & Hydrophobic (11) & Trp195, Leu254, Ala261, Gly265, Ala266, Gly268, Val269, Val271, Leu273, Ile275, Pro277 \\
\hline & Hydrophobic (10) & Arg203, Ser249, Ser255, Thr258, Glu262, Arg263, Ser270, Glu272, Lys274, Asp278 \\
\hline
\end{tabular}

all molecules in the EstE7 structure remain fully accessible to the substrate. Thus, the structure of EstE7 is similar to that of other dimeric HSL-homolog proteins.

\section{Conclusion}

Here, we reported the structural and biological analysis of the HSL-homolog EstE7. In the solution state, oligomeric states of EstE7 have differential molecular masses and occur as aggregated, dimer and monomer molecules. The results of this study indicate that the preferred oligomeric state of the EstE7 protein is the dimer formation. Based on the structure, this dimerization is stabilized by $\beta 8$-strands using salt bridges, hydrogen bonds and van der Waals interactions as in other dimerized HSLhomolog proteins. Thus, the biochemical and structural results presented here may follow the enzyme stability of the dimer formation. We anticipate that these results will provide useful information that enables a better understanding of the oligomeric state of HSL-homolog proteins.

Acknowledgments. We thank the beamline staff for assistance during data collection at beamline $6 \mathrm{C}$ of the Pohang Light Source, Korea. This work was supported by the Korea Science and Engineering Foundation [R01-2007-000-20072-0 (2010)]. K.H. Nam was supported by a Research Fellowship from the Brain Korea 21 program.

\section{References}

1. Osterlund, T. Eur. J. Biochem. 2001, 268, 1899-1907.

2. Yeaman, S. J.; Smith, G. M.; Jepson, C. A.; Wood, S. L.; Emmison, N. Advances in Enzyme Regulation 1994, 3434, 355-370.

3. Raclot, T.; Holm, C.; Langin, D. Biochim. Biophys. Acta 2001, 1532, 88-96.

4. Wei, Y.; Contreras, J. A.; Sheffield, P.; Osterlund, T.; Derewenda, U.; Kneusel, R. E.; Matern, U.; Holm, C.; Derewenda, Z. S. Nat. Struct. Biol. 1999, 6, 340-345.

5. De Simone, G.; Galdiero, S.; Manco, G.; Lang, D.; Rossi, M.; Pedone, C. J. Mol. Biol. 2000, 303, 761-771.

6. De Simone, G.; Menchise, V.; Manco, G.; Mandrich, L.; Sorren- tino, N.; Lang, D.; Rossi, M.; Pedone, C. J. Mol. Biol. 2001, 314, 507-518.

7. De Simone, G.; Menchise, V.; Alterio, V.; Mandrich, L.; Rossi, M.; Manco, G.; Pedone, C. J. Mol. Biol. 2004, 343, 137-146.

8. Mandrich, L.; Menchise, V.; Alterio, V.; De Simone, G.; Pedone, C.; Rossi, M.; Manco, G. Proteins 2008, 71, 1721-1731.

9. Nam, K. H.; Kim, M. Y.; Kim, S. J.; Priyadarshi, A.; Kwon, S. T.; Koo, B. S.; Yoon, S. H.; Hwang, K. Y. Proteins 2009, 74, 10361040.

10. Nam, K. H.; Kim, M. Y.; Kim, S. J.; Priyadarshi, A.; Lee, W. H.; Hwang, K. Y. Biochem. Biophys. Res. Commun. 2009, 379, 553556.

11. Byun, J. S.; Rhee, J. K.; Kim, N. D.; Yoon, J.; Kim, D. U.; Koh, E.; Oh, J. W.; Cho, H. S. BMC Struct. Biol. 2007, 7, 47.

12. Fernandez, L.; Beerthuyzen, M. M.; Brown, J.; Siezen, R. J.; Coolbear, T.; Holland, R.; Kuipers, O. P. Applied and Environmental Microbiology 2000, 66, 1360-1368.

13. Otwinowski, Z.; Minor, W. Macromolecular Crystallography 1997, 276, 307-326.

14. Vagin, A.; Teplyakov, A. Journal of Applied Crystallography 1997, 30, 1022-1025.

15. Winn, M. D.; Murshudov, G. N.; Papiz, M. Z. Methods Enzymol. 2003, 374, 300-321.

16. Vaguine, A. A.; Richelle, J.; Wodak, S. J. Acta Crystallographica Section D-Biological Crystallography 1999, 55, 191-205.

17. Emsley, P.; Cowtan, K. Acta Crystallographica. Section D-Biological Crystallography 2004, 60, 2126-2132.

18. Davis, I. W.; Leaver-Fay, A.; Chen, V. B.; Block, J. N.; Kapral, G. J.; Wang, X.; Murray, L. W.; Arendall, W. B.; Snoeyink, J.; Richardson, J. S.; Richardson, D. C. Nucleic Acids Res. 2007, 35, 375-383.

19. Dodson, E. J.; Winn, M.; Ralph, A. Methods Enzymol. 1997, 277, 620-633.

20. Brunger, A. T.; Adams, P. D.; Clore, G. M.; DeLano, W. L.; Gros, P.; Grosse-Kunstleve, R. W.; Jiang, J. S.; Kuszewski, J.; Nilges, M.; Pannu, N. S.; Read, R. J.; Rice, L. M.; Simonson, T.; Warren, G. L. Acta Crystallogr. D. Biol. Crystallogr. 1998, 54, 905-921.

21. Krissinel, E.; Henrick, K. J. Mol. Biol. 2007, 372, 774-797.

22. Holm, L.; Kaariainen, S.; Rosenstrom, P.; Schenkel, A. Bioinformatics 2008, 24, 2780-2781.

23. Shen, W. J.; Patel, S.; Hong, R.; Kraemer, F. B. Biochemistry 2000, 39, 2392-2398.

24. Shire, S. J.; Shahrokh, Z.; Liu, J. J. Pharm. Sci. 2004, 93, 13901402. 